\title{
Smart Design for Ships in a Smart Product Through-Life and Industry 4.0 Environment
}

\author{
Joo Hock ANG \\ Sembcorp Marine Ltd, Singapore; and \\ University of Glasgow, UK \\ joohock.ang@sembmarine.com
}

\author{
Cindy GOH \\ School of Engineering \\ University of Glasgow, UK \\ Cindy.Goh@glasgow.ac.uk
}

\author{
Yun LI \\ School of Engineering \\ University of Glasgow, UK \\ Yun.Li@glasgow.ac.uk
}

\begin{abstract}
As the world becomes more connected and customer's 'needs and wants' lean more towards bespoke products, the way they are designed and manufactured must adapt. Recognised as the main driver for the future of the manufacturing value chain, Industry 4.0 (i4) is rapidly gaining momentum worldwide. One of the key enablers of the i4 design model to achieve mass customisation manufactured at a mass production cost is the computational intelligence based Computer-Automated Design (CAutoD). This paper demonstrates how CAutoD realises the i4 concept for smart design of future ships and smart ships through-life. Following an overview of the i4 and CAutoD interface, a smart ship design technique is introduced to form an automated closed-loop approach to the entire ship design process. Then, key challenges and future directions on this roadmap are discussed. Lastly, a framework in which the concepts of morphing and free-form deformation are embedded into an evolutionary algorithm is developed to automate the design and optimisation process of the hull form.
\end{abstract}

Keywords-ship design; industry 4.0; computer-automated design; smart design; evolutionary algorithm

\section{INTRODUCTION}

The world is getting increasingly connected both in the virtual as well as physical space. At same time, the customer's 'needs and wants' is evolving, from products that are mass manufactured to those that are bespoke. The advent and popularisation of the internet in 1990s has helped to advance the interactions between people and systems in an unprecedented manner. Industry 4.0 (i4), a concept engineering 'fourth industrial revolution' that was recently introduced by the German government aims to revolutionise how products are built and manufactured [1]. It encompasses concepts from cyber-physical system, internet of things, big data, e.g. i4 supports design and manufacturing trends in moving towards higher creativity, lower cost, and better responses to customer needs while at the same time coming up with on-demand production of optimal and intelligent solutions [2].

CAutoD, commonly known as virtual rapid prototyping is an extension of traditional Computer-Aided Design (CAD) [3]. It makes use of biologically-inspired machine learning techniques such as an evolutionary algorithm (EA) to intelligently search and evaluate the design space for innovative and optimal solutions. Coupled with powerful

This research is funded by Economic Development Board (EDB) of Singapore and Sembcorp Marine Ltd. under Industrial Postgraduate Programme (IPP) grant no: COY-15-IPP/140002. evaluation tools, this forms a close loop to fully automate the design process.

Shipbuilding is a highly complicated process involving many disciplines and stakeholders, often situated in multiple geographical locations. It is also an expensive and lengthy process with new vessels costing several tens to hundreds of millions and requires many man-months to build. Recent fuel price volatility and increase in stringent environmental regulations are changing the way vessels are design and built. These macro-level factors and shorter time-to-market pressures have called for vessels to be more energy efficient and constructed in the most cost effective manner. Ship design typically consists of four phases - concept design, contract design, preliminary design and detail design. These design processes are highly iterative - it takes many man-months to redesign and update before the final design is considered 'optimal'. Traditionally, design improvements or design optimisations are carried out manually with ship designers relying much on their experience and conventional CAD and Simulation-Based Design (SBD) tools. As computational methods and high performance computing (HPC) advances, the use of more high-end optimisation (i.e. EA) and performance evaluation procedure (i.e. Computational Fluid Dynamic (CFD)) are becoming more prevalent in the ship design process. However, the adoption of these advance techniques in the marine industry is somehow limited due to lack of a "closeloop' approach where design can be fully automated with little or no user dependencies.

To add to this, the multitudes of competing design and performance objectives proved to be a showstopper for conventional design methods to be fully automated. Also, with increased sophistication in advance equipment and monitoring systems installed on-board the vessel as well as increase awareness and new regulations in environment protection, product through-life becomes an important topic to be considered during design stage. To keep abreast, shipyards are constantly looking for new ways to leverage on the state-ofthe-art technology. i4, which is set to revolutionise the way future products are design seem a promising candidate to fit the bill. In this paper, we explore how CAutoD can be integrated into the entire i4 concept for smart ship design. This paper is organised into 5 sections. Firstly, an overview of Industry 4.0 and CAutoD is given in the Section II. In Section III, we look at smart ship design - how CAutoD can be linked with i4 to form an automated close-loop design process. In this section, we also highlight the challenges and possible future directions 
of smart ship design. Following this, a specific aspect of ship design, i.e. hull form design and optimisation (HFDO) is detailed in Section IV. Specifically, we embed the concepts of morphing and free-form deformation into an EA to automate the design and optimisation process of the hull form. Finally, the paper will be summarised with concluding remarks in Section V.

\section{INDUSTRY 4.0 AND COMPUTER-AUTOMATED DESIGN}

\section{A. Industry 4.0 overview}

Building on the basis of cyber-physical production systems (CPPS) which merges the real and virtual space [4], Industry 4.0 (i4) is a collaborative network which combines internet of things, services, data and people. It first originated from a high tech strategy project from the German government in 2011 [5] and is generally referred to as the fourth industrial revolution as depicted in Figure 1. i4, when successfully implemented, can transform traditional production from scattered and isolated cells to fully integrated, automated and optimised production flow for greater efficiencies and closer manufacturing relationships between humans (suppliers, producers, customers) as well as machines [1]. As such, it is widely recognised as the future of productivity and growth in manufacturing sectors or 'factory of the future.'

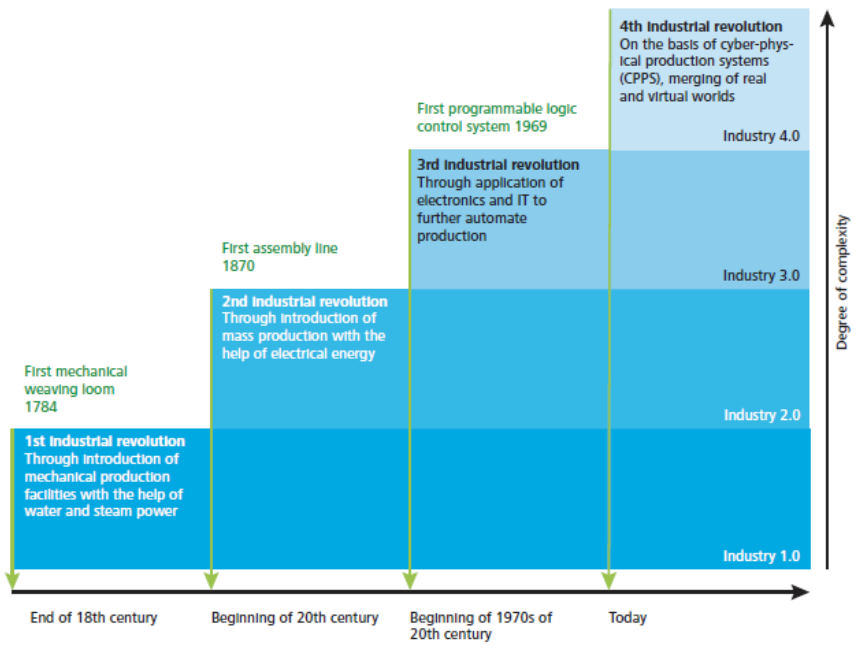

Fig. 1. Definition of Industry 4.0 [4]

There are many benefits in applying i4 for product design and manufacturing companies. First, it opens up new pathways to integrate customer needs and preferences into the product development process. It also offers new ways of creating value and novel business models [2], as well as improving resource efficiency. Through close networking between resources and products over real time optimised network, better communications between machineries and other parts of the system can be achieved such that waste associated with the development process is significantly reduced. A key strength of i4 is flexibility - any system built on the i4 principles should be sufficiently nimble to respond flexibly to last minute changes in production, disruptions and failures. In addition, i4 facilitates optimised design and decision making through end- to-end transparency in the manufacturing process [2]. Nonetheless, for i4 to be successful it is necessary to revamp and redevelop current systems and infrastructure across entire value chain.

- While i4 is relatively new, many associated concepts and technologies have been built and this is set to continue to increase. Figure 2 provides an overview of the i4 platform and its related technologies. These include: Smart factory - Smart Factory can be defined as a factory where cyber physical system (CPS) communicate over the Internet of things (IoT) and assist people and machines in the execution of their tasks [6]. Through CPS, machineries, storage systems and production facilities can autonomously exchange information, triggering actions and controlling each other independently [7]. Horizontal and vertical system integration allows enterprise view of data and network of systems, collaboration across different disciplines and value chains. It integrates different levels such as physical world dimensions, multi-domain methods, scheduling models, design views, software tools, physical components, e.g. [2]. Smart factory enables production to be customer specific and highly individualised yet still can be manufactured profitably by maintaining efficiency obtained from automated production [4]. Other components of smart factory include additive manufacturing using $3 \mathrm{D}$ printing, augmented reality which supports virtual training and on-the-job instructions and autonomous robots that are deployed in smart assembly lines.

- Internet - There are many terms that describe the application of internet to i4, that includes Internet of everything (IoE), Internet of Things (IoT), Internet of People (IoP), Internet of Service (IoS), and most recently, 'Internet + '. Internet of Everything (IoE) is a broad term that refers to devices and consumer products connected to the Internet and outfitted with expanded digital features. Internet of Things (IoT) is the network of physical objects or "things" embedded with electronics, software, sensors, and network connectivity, which enables these objects to collect and exchange data [8]. Using IoT devices and embedded computing sensors, it allows real time response for more predictive maintenance with condition monitoring [4].

- Business informatics - Business informatics consists of a range of technologies that supports communication, storage, analysis of data and information that are useful across different business functions. Through cloud computing, it allows real time data transfer and scalability, industrial monitoring and control system. It also provides infrastructure, platform and software as services which enable convenient, on-demand network access to a shared pool of configurable computing resources [9]. Big data analytics is applied here to ensure data availability for targeted innovation, marketing and decision making. Data mining can then extract useful knowledge obtained from big data and use for various business process such as improving 
design or manufacturing processes, adjust sales and marketing strategies, e.g. On the other hand, machine learning methods can be applied here for predictive analytics or predictive modelling so as to identify risk and future opportunities. Product life management (PLM) can help to manage the entire lifecycle of a product efficiently and cost-effectively, right from idea conception, design and manufacture, to service and subsequent disposal.

- Smart design - Smart design consist of a combination of techniques and tools which aid smart product design, including computational intelligence, SBD, design automation, e.g. In this case, by automating the simulation of product scenario using SBD and computational intelligence techniques, it can helps in the rapid testing and development of innovative design, which in turn translate to better products and revenue.

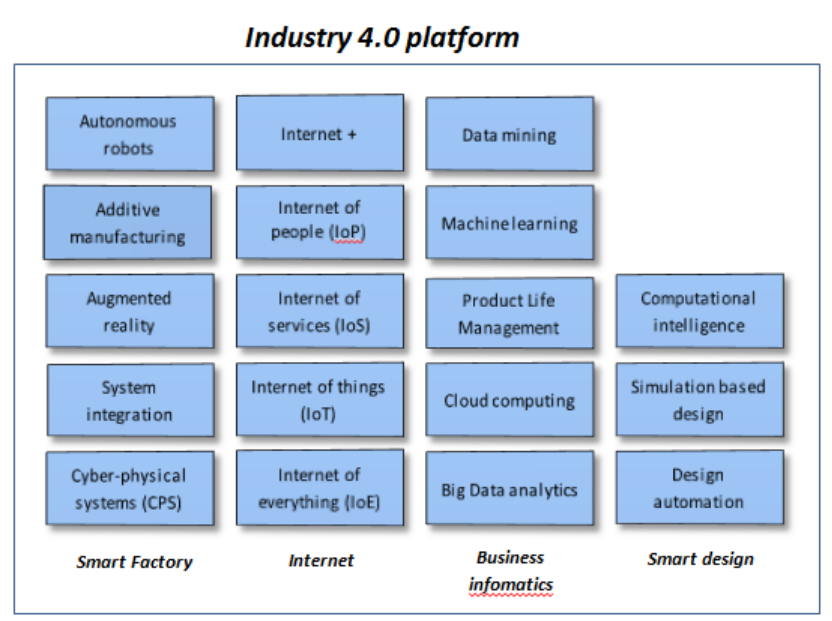

Fig. 2. i4 platform and associated concepts

\section{B. Computer-automated design}

To stay competitive, companies are always looking at ways to reduce cost and improve the quality of their products. To achieve this, traditional physical prototyping is not anymore the only option for product development but can be complimented with virtual prototyping which aims to meet multiple objectives such as maximum output, reduced weight, cost effectiveness, e.g. Virtual prototyping make use of SBD software to simulate and validate the performance of a design concept before an actual physical prototype is build. It can also be used to tweak and improve existing designs. Through virtual prototyping, engineers have now the luxury of exploring many design alternatives before investing the time and money to construct physical prototypes. An obvious benefit of this method is reduced time-to-market, cost and products with higher level of performance and reliability.

CAutoD is a design automation concept which combines $\mathrm{CAD}$, computational intelligence and virtual prototyping. It goes a step further than traditional virtual prototyping where existing designs can be optimised and create innovative designs. In the context of any new product design, this would involve finding the best design within known range (i.e. optimisation) and/or finding a new and better design through learning (i.e. creation) [3]. This can be formulated into a search problem and solved using computational intelligence methods such as Genetic Algorithm (GA), Particle Swarm Optimisation (PSO), and Ant Colony Optimisation (ACO), e.g. These biologically inspired search methods mimic the natural process of nature evolution. They make use of past trial information in an intelligent manner similar to that of a human designer [3]. Figure 3 shows how CAutoD forms the close loop for product design by coupling computational intelligence methods and performance evaluation. By removing the need for human intervention, the search for optimum design is performed by intelligent computer algorithms, which in turns significantly reduce the design time and user dependencies.

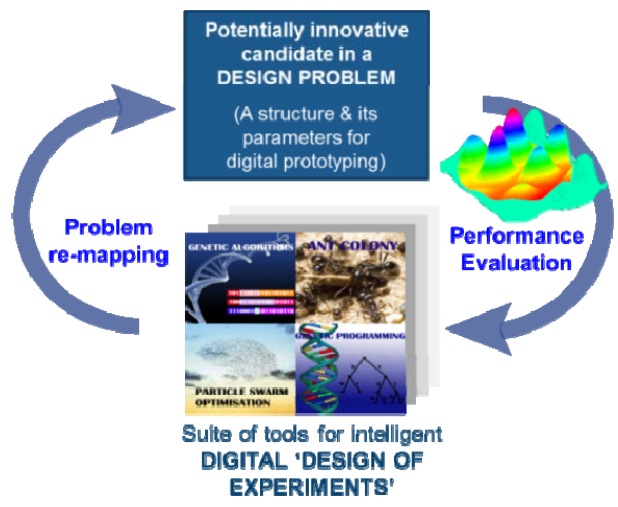

Fig. 3. Computer-automated design

\section{Integration of CAutoD to i4}

As described in the earlier section, smart design is an important component in i4 which includes concepts such as computational intelligence, SBD, e.g. CAutoD possess similar characteristics to smart design in $\mathrm{i} 4$ such as computational intelligence as well as design automation. In addition, CAutoD incorporates virtual prototyping and design optimisation which can be used to complement smart design in i4. On top of improving the overall design process, CAutoD can also support the development of other i4 concepts such as smart factory, business analytics, e.g. In table 1, we provide a correspondence matrix showing relations between i4 smart design and CAutoD.

TABLE I. CORRESPONDENCE MATRIX BETWEEN I4 SMART DESIGN AND CAUTOD

\begin{tabular}{|l|c|c|}
\hline \multicolumn{1}{|c|}{ Design concepts } & $\begin{array}{c}\text { Smart } \\
\text { design }\end{array}$ & CAutoD \\
\hline Simulation based design & $\sqrt{ }$ & $\sqrt{ }$ \\
\hline Computational intelligence & $\sqrt{ }$ & $\sqrt{ }$ \\
\hline Design automation & $\sqrt{ }$ & $\sqrt{ }$ \\
\hline Machine learning & $\sqrt{ }$ & \\
\hline Virtual prototyping & & $\sqrt{ }$ \\
\hline Design optimisation & & $\sqrt{ }$ \\
\hline
\end{tabular}




\section{SMART SHIP DESIGN}

\section{A. Smart ship design concept}

Smart ship design is termed in this paper as an intelligent ship design process, which possesses interconnected features that has the potential to elevate and improve the entire shipbuilding process using computational intelligence techniques. It is important to note the distinction between 'smart design' of ships and design of 'smart ships'. The former represents smart ways of designing ships, i.e. more efficient and productive ways to design, which is the focus of this paper, while the latter represents designing ships which are smart, i.e. ships with built-in intelligent capabilities.

In this paper, our concept of smart ship design is based on i4, which in turn supports the development of i4 for ship design application. By virtue of smart ship design, the design process should be fully digitalised and connected in a network which enable self-gathering and analysis of critical information obtained from manufacturing, in-service operations, big data, e.g., which in turn provide automatic feedback link to influence the new design process. It can also include other features suggested as follows:

- More intelligent and robust design - A good design depends greatly on the expertise of the designer and in the face of aging workforce around the world; knowledge capturing becomes an important topic both for design and construction. Through CAutoD, explicit knowledge and important information can be captured automatically and present as a useful tool for new designer. Using virtual reality, clash check and design improvement can be carried out in 3D simulated environment before construction, which will help to reduce reworks due to error. Through data mining and machine learning, the system can automatically translate useful data back into the design and even adjust the design parameters to suit any new requirements.

- System integration and network connectivity - Design integration between different disciplines such as structural, mechanical, piping, electrical, e.g. Design process are hereby closely connected to other processes such as planning and construction whereby allowing real time design changes depending on customer demand and production output.

- Benchmark best design practices and product life cycle - Through big data and data analytics, best design practices can be collected both internally and externally, compared and incorporate back into the design process. Using product life management, ship's actual operating profile up to decommissioning can be captured, stored and considered as part of design for product life-cycle.

Using concepts based on i4 and CAutoD as well as features identified above, we conceptualise an example of smart ship design application by connecting CAutoD with cyber-physical system and business informatics to form a closed design loop, as illustrated in figure 4 and further explained as follows:

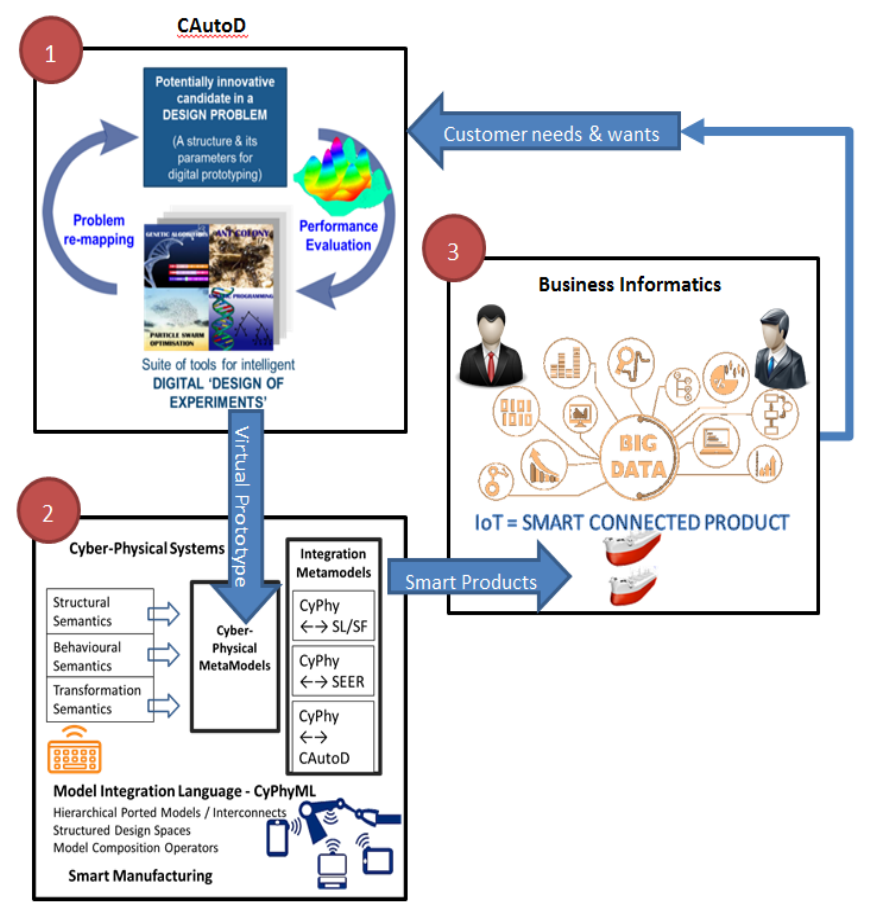

Fig. 4. Smart ship design

1) CAutoD: The design process first start from taking inputs from business informatics where customer needs and possible regulatory requirements are predicted and incorporates as design criteria into the CAutoD process. It also takes in other design criteria which can come from actual operation performance of similar vessel types such as weather condition, power and fuel consumption pattern, e.g. In this process, the designer can also adjust its design based on customer or construction feedback such as changes in owner's requirement and site clashes. The entire design process is developed based on single 3D model put on cloud where various stakeholders such as owner, classification bodies and engineers from different disciplines can access, modify and update using the same model [10], whereby eliminating unnecessary delays and error due to human error or engineering details that are lost in translation. Using virtual reality tools, engineers can improve the design or check for potential clashes before construction. High performance computers are highly utilised in conjunction with CAutoD for high definition performance evaluation and simulations such as CFD.

2) Smart manufacturing/ Cyber-physical system: Once sufficiently optimum design are obtained, they are fed into cyber-physical model where production system and factory machineries can communicates with each other via a network, commonly known as Cyber-physical production systems (CPPS). These interconnected systems and machines will inturn translate the designs into fabricated structures in the shop floor, which are constructed based on latest design and customer requirements. It measures and takes into consideration the productivity, wastages, e.g. By taking into 
consideration of the latest stock level in the inventory as well as manpower deployment, it is capable to adjust the production process, output and schedule accordingly. Condition monitoring enables planned maintenance to be carried out timely before particular machines breaks down and affects the production schedule.

3) Business informatics: Production output such as schedule, manpower productivity and material usage are then fed into business informatics. Through big data, useful information are collected, filtered and analysis so it can be disseminate back into various processes such as design, sales, production, e.g. Through advance sensors and monitoring devices, in-service ship performance such as power and fuel consumption and weather can be collect remotely through cloud and feedback to process (1) to improve the design. Using Product life cycle management, entire lifecycle of the vessel can also be monitored and managed more efficiently from concept, design and construction, in-service to subsequent decommissioned and scrapped. Centralised data and document management allows the vast amount of documents and drawings generated across the project lifecycle to be stored and made accessible internally within shipyard as well as external organisation such as owners and regulatory bodies.

\section{B. Key challenges for smart ship design}

In order for smart ship design to be realised and applied to actual ship design, there remains many challenges. While developments of $i 4$ are still on-going, we list down some key challenges that are specific to the application of i4 to shipbuilding and smart ship design, which are suggested as follows:

- Customised design - For new shipbuilding, ships is increasingly customised to specific needs of customers who are mainly ship owners and/or ship operators. These customisation includes adding of additional equipment (monitoring system, scrubber, e,g.) which requires the rearrangement of compartment space and related systems or changing of build materials (carbon steel pipe to duplex stainless steel) for ease of maintenance during operation, e.g. As these customisation usually deviate from 'standard' design and manufacturing processes, it causes disruption to the 'normal' work methodology and additional manpower would need to be allocated to work on these deviations, which inevitably increase the overall cost and project schedule.

- Communication of different systems and design tools This is poised to be the biggest obstacle for successful transition from traditional ship design to truly smart ship design. More often, most shipyard and design firm use many different software tools for different functions such as hull modelling, stability, structure analysis, hydrodynamic analysis, e.g. [11]. With so many stakeholders situated in different geographical locations, each using their own proprietary and commercial design software, communication between these different systems and software becomes the top most priority to overcome. With more automation and sophisticated Computer Numerical Control (CNC) machines deployed in production floor and each machines using different software, there is also a need to align the system architecture between production and design system.

- Product life cycle - As part of smart ship design features, it needs to consider entire life phase perspective from product development, to production, delivery, in-service operation, maintenance, remanufacturing or scrap. However, monitoring and implementation of this perspective is extremely challenging. Key challenge includes system and management integration, lack of collaboration and lifecycle tracking mechanism [12], e.g.

- IT infrastructure and cyber risk - Most companies requires good IT infrastructure to be in place in order to support i4/ smart design systems such as internet of everything, big data, cloud computing, high performance computer, e.g. With more internet-enabled devices that are connected to web, it inherently creates more risk to business from cyber-attack and other IT disasters which may threatens business continuity. More developments on IT security are therefore needed in order to increase the confidence of user.

- Manpower resource - For companies to apply i4 and smart ship design, it requires a large pool of highly skilled IT professionals, designers and CFD engineers in order to design, operates and maintain these highly integrated and sophisticated systems and software tools. Unfortunately, these skilled personnel are however not easy to find and expensive to employ.

- Limitation of current i4 supporting mechanism Current mechanism used in supporting i4 may not be designed and sufficient to support modern data complexity and huge data set. Some examples of current limitation include wireless connection speed and system integration.

- Decentralised system - One main barrier as identified in [2] is decentralised systems for allocating hardware and software resources to individual workstation, which had been designed to operate with limited knowledge of complete system. However, decentralisation is becoming a trend in business environment as it has its benefits of forming self-organising emergent system, with self-adaption, self-management and self-diagnosis. It allows control of more complex systems although global optimal performance cannot be guaranteed [2].

- General - With so many different systems and additional investment required, it is extremely difficult to quantify the economic benefit or return of investment (ROI) in the application of i4 to smart ship design. 


\section{Opportunities and future directions for smart ship design}

With the challenges stated above, we suggest some recommendations for successful implementation of i4 and smart ship design, listed as follow:

- Customised design - One of the main aims of i4 is to achieve mass customisation manufactured at a mass production cost. Through i4 concepts such as CAutoD and cyber-physical systems, this can be realised as the various business processes becomes fully digitalised and connected, ad-hoc customisation or even last minute changes can be effected immediately right from design to production floor. Barring any major cost increases due to the changes (upgrading material to higher specification, e.g.), these additional cost of separate designers or production workers time to work on the customisation can be offset substantially through automation and increased efficiency so that the overall cost and entire schedule are not affected.

- Communication of different systems and design tools OpenMETA [2] is a tool integration framework that incorporates a network of model transformations that include model for individual tools and integrate modelbased design flows. Advantage of this system is its ability to address scalability and system wide optimisation, managing the evolution of data, data presentations and use of service integration over time [2]. This is especially useful in ship design and virtual prototyping where different $\mathrm{CAD}$ tools are used for hull modelling, hydrodynamic analysis and simulations. By integrating the different software, interchangeability and interoperability of the model can be further enhanced.

- Smart products through-life - In order to consider product life cycle through smart product concept, it was recommended in [12] to integrate through-life product intelligence within smart products, with intelligent manufacturing systems in order to optimise entire value chain. Potential solution includes integrated design, intelligent systems, artificially intelligent products/ systems, big data analytics, e.g., which are closely associated to i4. In smart ship design context, monitoring and control will have to start straight from contract award by ship owners to ship construction by shipyard, approval by regulatory bodies, to subsequent operation and maintenance by ship management company, to subsequent decommission or scrap by ship owners. Taking average lifespan of 25 years for typical sea-going vessel, it would requires a single monitoring system which are highly connected in order to track such high data volume handled by all parties across the entire life-cycle. It is therefore important to further develop on i4, in particular on the concept of smart product through life-cycle that are customised to ship application and apply to smart ship design.

- IT infrastructure and cyber risk - As designs analysis requirement becomes more complicated and move towards high definition, development of Graphical processing units (GPU) and exa-scale computing can helps to reduce the computation requirement and processing time. However, software tools must also be developed in parallel to fully capitalised on the upgraded performance of IT hardware. In order to build confidence for new framework, companies can implement security protocol and develop internal IT disaster recovery plan to ensure business continuity.

- Manpower resource - In order to overcome the shortage of skilled IT and CFD personnel, companies can invest more on training and upgrading of existing staff in order for them to be more competent to apply i4 techniques and CFD simulations. Technical skills that are useful for successful i4 implementation may includes programming, data management, system integration, IT security and other 'soft' skills such as risk management, behaviour development, e.g.

- Current i4 supporting mechanism limitations - In order to overcome the limitation of current supporting mechanism for i4, it is important to develop new techniques or algorithms that are capable to capture the complexity of modern data and scalable enough to process huge datasets in parallelised and fully decentralised fashion [13]. For example, to overcome limitation of wireless connection for implementing i4 systems which requires transfer of huge dataset, there is a new generation wireless connection $5 \mathrm{G}$ which is under formal research and development which may help to resolve current limitation [2].

- General - In order to fully capitalise entire i4 concept, development should extend beyond smart manufacturing and smart design and move into smart sales and marketing, business forecast, for example. By introducing more smart features and concept into i4 platform through development of relevant or new technologies, it will further enhance and expedite the realisation of i4 in marine industry and smart ship design.

\section{Hull Form Design OptimisATION}

\section{A. Hull form design optimisation framework}

In order to overcome the manual design process and fully exploit the benefit of advance optimisation and high definition performance evaluation techniques, important functions such as design automation, efficient shape manipulation and robust optimisation needs to be developed and incorporated into the ship design process. Using concepts of CAutoD, a new framework - hull form design optimisation (HFDO) is herein presented as an improved version from [14]. The new framework will integrate the merits of EA, geometry manipulations and variable performance evaluation techniques into a fully automated ship design optimisation solution as illustrated in figure 5 . 


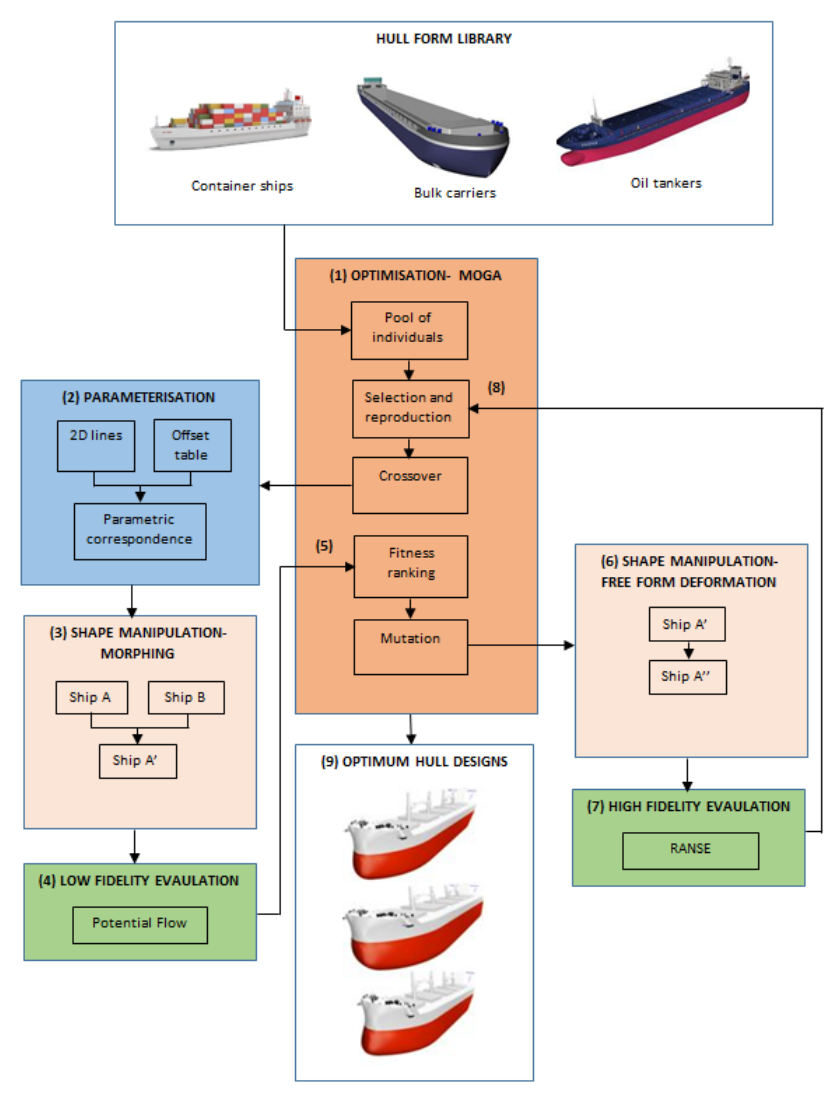

Fig. 5. Hull form design optimisation framework

Specifically, the HFDO consists of 9 stages altogether as detailed below:

Hull form library: In ship design, most designers either start from using existing design or from scratch. The advantage of using existing design is due to proven and matured design which guarantees the performance as compared to starting from scratch, which will take much longer time although it may produce more novel designs. This library contains a collection of hull forms for different ships which are completed earlier in the ship design office or shipyard. The hull data can be compiled based on offset table or lines plan. As different ship type are designed with different objective function, it is useful to sort them according to their ship type such as container vessel, bulk carries or oil tanker, e.g. and objective function such as reduce resistance, improve sea-keeping, increase stability, e.g.

1) Optimisation: This is the first and most important step of the entire framework which explores the design search space and automates the entire design optimisation process. Using EAs such as Multi-objective Genetic Algorithm (MOGA), it can be used to navigate efficiently multidimensional, large and challenging search space such as hull form to produce globally optimal non-dominated solutions [14]. In this process, the designer has the option to select from a few existing hull form designs from the hull from library, which are closest to the new design requirement and use to form a pool of individual design. The first pool of design will then be selected according to the objective function and design variables that are defined by designer for geometry deformation and evaluation.

2) Parameterisation: Before the crossover or morphing process starts, it is required to parameterise and corresponds the hull geometry so that both original hull form will share the same number of vertices. One possible way to automatically identify and select same number of feature or control points within a ship curve is through the shortest path method.

3) Crossover- Morphing: With both hull forms parameterised, the original hull forms (parents) can then be transformed using 3D surface morphing and produce a range of intermediate new designs (child). These designs will be used as the first set of candidate design solutions for evaluation.

4) Performance evaluation (low-fidelity): The hydrodynamic performance of all candidate design solutions generated from morphed designs in (3) will first be assessed using coarse resolution or low-fidelity computational fluid dynamic (CFD) simulation such as potential flow, which can provide very fast evaluation results even though it is not very accurate for measuring turbulence flow in way of stern area as well as hull appendages.

5) Fitness ranking: With the results are obtained from (4), these candidate design solutions (transformed hull form) will be ranked based on their fitness level (performance) within the optimiser before passing on to next transformation stage.

6) Mutation - Free-form deformation: In this stage, minor transformation or localised modification will be carried out here to inject novelty into the design. It also allows the flexibility of designer to input his/her preferred design features based on past experiences into the design optimisation process. Examples include modifying the bulbous bow to be sharper or stern area to be fuller, which will provide a new design dimension into the design optimisation process.

7) Performance evaluation (high-fidelity): Once the enhanced design is obtained from (6), the final candidate design solutions will be evaluated using high resolution or high-fidelity evaluation such as RANSE method. RANSE is a highly accurate CFD method used for ship evaluation within the marine industry but it is highly computation expensive. One way to overcome is through high performance computers and parallel computing [11].

8) Selection and reproduction: These evaluated final candidate solutions are once again mixed together with pool of new individuals taken from hull form library to re-model and re-assessed within the optimisation framework. This process will continue to iterate and terminate once the design criteria defined by the designer are met.

9) Optimum hull design: Finally, a range of nondominated optimal solutions will be identified as part of HFDO output. The designer are now presented with a selection of optimised hull forms with detailed performance results within a short span of time, which he/she can select based on project's design criteria. 


\section{B. Benefit of $H F D O$}

There are many benefits in using HFDO, both as a standalone framework or in conjunction with i4. The advantages of this new framework are as follows:

- Automated process - As mentioned earlier, one of the main hurdle in product design is the lack of 'closedloop' approach in conventional design methods. By using CAutoD concepts which provide the link to close the loop, the entire design and optimisation process can be fully automated without user intervention, which will vastly improve the design efficiency as well as attaining better designs with superior performance.

- Comprehensive and well balanced tool - This framework utilises both low-fidelity (potential flow) and high-fidelity (RANSE) evaluation methodologies, which helps to balance the 'needs and wants' between computation speed and high quality simulations. It is seamlessly connected with different shape manipulation methods that are coupled within computation intelligence technique such as MOGA.

- Feasible designs - There are many advantages using morphing method for hull shape transformation. Firstly, the 'morphed' design is based on matured designs which guarantee the performance without having to start the entire hull form design from scratch. Second, a new hull form design can be generated very quickly using morphing method which ensure all generated designs are feasible and reduces the time significantly for the entire optimisation process.

- Complementary to smart ship design - HFDO can be coupled to smart ship design and complement each other in many ways. Firstly, by linking hull form library to business informatics, a large variety of hull form data can be collected through big data which increases the individuals in the selection pool size within HFDO exponentially. Actual ship performance data collected from business informatics through advance monitoring system can also influence or adjust the design criteria set under optimiser (MOGA), which will result in better designs due to life-cycle consideration. Overall, by seamless connection with other key process in smart ship design (business informatics, e.g.), HFDO can serve as an efficient virtual prototyping tool in fulfilling the goal of smart ship design.

\section{CONCLUSION}

With the world becoming more connected and customised, design and manufacture are evolving to satisfy and also capitalise on the trend. Industry 4.0 addresses this using a cyber-physical system in the future. With CAutoD, intelligent virtual prototyping helps i4 to automate and optimise design for mass customisation. This allows the realisation of smart ship designs which possess interconnected features that have the potential to elevate and improve the entire shipbuilding process. However, the development and implementation of smart ship design is limited by different systems, IT infrastructure and limitation of existing design tools. Several key recommendations are proposed to overcome some of these challenges, including implementation of tool integration framework and smart products through-life. Based on CAutoD, a new framework, HFDO, has been developed in this paper to embed morphing, free-form deformation and both low and high fidelity evaluation processes into an EA. It has automated part of the design and the optimisation process of the hull form so far. Based on this case study, we believe smart ship design and HFDO can help revolutionise the entire shipbuilding process in the future and eventually be adopted by the marine industry in the upcoming intelligent Industry 4.0 era.

\section{REFERENCES}

[1] Michael Rüßmann et al, "Industry 4.0: The Future of Productivity and Growth in Manufacturing Industries, " BCG online article, April 2015.

[2] Alfredo Alan Flores Saldivar et al, "Industry 4.0 with Cyber-Physical Integration: A Design and Manufacture Perspective," in Proceedings of the 21st International Conference on Automation \& Computing, Glasgow, 2015.

[3] Li, Y., et al., CAutoCSD - "Evolutionary search and optimisation enabled computer automated control system design", International Journal of Automation and Computing, 1(1). 76-88, 2004.

[4] "Industry 4.0: Challenges and solutions for the digital transformation and use of exponential technologies," Deloitte report, Oct 2014.

[5] Kagermann, H., W. Wahlster and J. Helbig, "Recommendations for Implementing the Strategic Initiative INDUSTRIE 4.0", Acatech National Academy of Science and Engineering (3), Apr 2013.

[6] Hermann Mario, Pentek Tobias, Otto Boris, "Design Principles for Industrie 4.0 Scenarios: A Literature Review," Technische Universität Dortmund, working paper no 01/ 2015.

[7] K.D. Kim and P.R. Kumar, "Cyber-Physical Systems: A Perspective at the Centennial", Proc. IEEE, 100, May, 1287-1308, 2012.

[8] Posada J. et al., "Visual Computing as a Key Enabling Technology for Industrie 4.0 and Industrial Internet", IEEE Computer Graphics and Applications, 26-40, March 2015.

[9] "Strategic R\&D Opportunities for 21st Century Cyber-Physical Systems, Technology, "N. I. o. S. a., Foundations for Innovation in Cyber-Physical Systems Workshop(6), 2013.

[10] Jeff Bashir, C.H., "How Intergraph SmartYard leverages the engineering design basis to improve work processes across the project life cycle, " in International Conference on Computer Application in Shipbuilding, Bremen, 2015.

[11] Ang, J.H., C. Goh, and Y. Li, "Key Challenges and Opportunities in Hull Form Design Optimisation for Marine and Offshore Applications, "in 21st International Conference on Automation \& Computing, Glasgow, 2015.

[12] Tijana Vuletic, "Workshop Notes: Smart Products Through-Life Workshop No 1", 29th October 2015.

[13] Boyd, S., N. Parikh, E. Chu, B. Peleato and J. Eckstein, "Distributed Optimization and Statistical Learning Alternating Direction Method of Multipliers, "in Foundations and Trends in Machine Learning, 2010.

[14] J.H. Ang, C. Goh, Y. Li, "Hull Form Design Optimisation for Improved Efficiency and Hydrodynamic Performance of 'Ship-Shaped' Offshore Vessels, "in International Conference on Computer Applications in Shipbuilding, Bremen, 2015. 